\title{
Compartmentalizing an SIR Model of $n$ Susceptibility Classes
}

$\begin{array}{ll}\text { Author: } & \text { Taliha Nadeem } \\ & \text { Undergraduate Student } \\ & \text { Benedictine University } \\ & \text { 3549 Hidden Fawn Drive } \\ & \text { Elgin, IL 60124 } \\ & \text { 630-664-2884 } \\ & \text { nadeem.taliha@gmail.com (preferred) } \\ & \text { zuhaa_nadeem@ben.edu } \\ & \text { Rachel Nicinski } \\ \text { Undergraduate Student } & \text { Benedictine University } \\ & \text { 889 Fieldside Lane } \\ & \text { Aurora, IL 60504 } \\ & \text { 630-423-1290 } \\ & \text { rachelnicinski@gmail.com (preferred) } \\ & \text { writersessence@gmail.com } \\ & \\ & \text { Dr. Anthony DeLegge } \\ & \text { Assistant Professor of Mathematics } \\ & \text { Benedictine University } \\ & \text { 5700 College Road } \\ & \text { Lisle, IL 60532 } \\ \text { 630-779-3655 } \\ \text { adelegge@ben.edu }\end{array}$




\begin{abstract}
The purpose of this research is to provide a solution to regulate environmental diseases when the spread of the disease is partly based on how susceptible different groups of individuals are. This study will monitor the effects a disease has on a population with groups of different susceptibility through the administration of vaccines: a point vaccination scheme (PVS) and an interval vaccination scheme (IVS). A PVS entails the instantaneous vaccination of individuals by selectively distributing vaccinations at one point in time, while an IVS is a more realistic vaccination method administered over an interval of time. Both schemes are exclusively administered to individuals classified as having the highest susceptibility to disease. The disease itself will be explored through the use of an $S I R$ model of $n$ susceptibility classes, a model which studies a disease in relation to susceptible, infected, and removed individuals.

To further study the disease, this same model will be modified through the incorporation of differential delay equations, denoting a regaining of susceptibility. These incorporations surface as a reflection of reality: in several diseases, it has been observed that vaccinated individuals or individuals with immunity to the original strain of a disease can regain susceptibility to a mutated strain, such as the common flu whose strains undergo constant mutation. Modification of the disease allows for recovering individuals to contract a viral variant.
\end{abstract}

\title{
INTRODUCTION
}

Out of the many that arise from environmental sources, infectious diseases are one of the leading causes of human deaths. Some of the deadliest infectious diseases include respiratory infections, malaria, HIV/AIDS, diarrheal diseases, tuberculosis, and neglected tropical diseases - such as rabies and leprosy [10]. In any given population, there are certain aspects of each age group that can dictate vulnerability: children with an underdeveloped immune system may be highly susceptible to certain disease, adults may begin to express inherited traits that interfere with their health, and the elderly may have a deteriorated capacity for health. Independent of age, hygienic habits may also contribute to an individual's susceptibility to disease; say, if someone hardly ever washes his/her hands. Also, others may be more susceptible to certain diseases because of malnutrition or the medications they may take.

To study the behavior of an epidemic as it affects individuals of different susceptibilities, an SIR model of $n$ susceptibility classes will be used. The overall goal of this study is to see what would happen to a disease if resources were limited, and only the most highly susceptible individuals could receive a vaccine against the disease. Ideally, this would limit the disease spread so much that it would eventually stop altogether, even though not everyone received the vaccine; we will investigate the conditions for when this occurs.

It is important to note that the terms "groups" and "classes" will be used interchangeably throughout the paper.

\section{AN SIR MODEL OF $\boldsymbol{n}$ SUSCEPTIBILITY CLASSES}

The standard SIR model presented by Kermack and McKendrick in 1927 [1], as shown below, denotes dynamic populations of susceptible, infected, and removed individuals with the corresponding differential equations, where $S, I$, and $R$ represent the susceptible, infected, and removed individuals, respectively:

$$
\begin{gathered}
S^{\prime}=-b S I, \\
I^{\prime}=b S I-\alpha I, \\
R^{\prime}=\alpha I .
\end{gathered}
$$

Susceptible individuals are those individuals who are not currently sick but can become sick; infected individuals are those who are currently sick and can infect susceptibles; removed individuals are those who have been taken out from the infective class because they can no longer become sick due to acquired immunity, quarantine, or death.

This model defines both the rate of infection that transmutes susceptible individuals into infectious ones and the rate of recovery that rejuvenates the infected individuals and - consequentially - places them in the removed group. The infection rate and recovery rate are respectively notated by $b S I$ and $\alpha I . b$ is a combination of the contact rate between the susceptibility and infectious classes and the probability of a susceptible individual getting infected when such a contact occurs. $\alpha$ represents how quickly infected individuals develop 
immunity, become quarantined, or die from the disease. Note here that $1 / \alpha$ is the average length of time an infected individual is infective, and, by definition, both $b$ and $\alpha$ are positive.

By modifying Kermack and McKendrick's model (1), an SIR model of $n$ susceptibility classes can be derived:

$$
\begin{gathered}
S_{1}^{\prime}=-b_{1} S_{1} I+p_{1} \beta-\mu S_{1}, \\
S_{2}^{\prime}=-b_{2} S_{2} I+p_{2} \beta-\mu S_{2}, \\
S_{3}^{\prime}=-b_{3} S_{3} I+p_{3} \beta-\mu S_{3}, \\
\cdots \\
S_{n}^{\prime}=-b_{n} S_{n} I+p_{n} \beta-\mu S_{n}, \\
I^{\prime}=\left(\sum_{k=1}^{n} b_{k} S_{k}-\alpha-\mu\right) I, \\
R^{\prime}=\alpha I+p_{R} \beta-\mu R .
\end{gathered}
$$

Due to the inclusion of a modified infection rate constant, $b_{\mathrm{i}}$, an SIR model of $n$ susceptibility classes gives the freedom to include differing levels of susceptibility. We denote the different susceptibility groups as $S_{1}$ (the highest susceptibility), $S_{2}$, and so on to $S_{n}$ (the lowest susceptibility). I will remain the infected group and $R$ will remain as the removed group. As the individuals in the $S_{1}$ class suffer the highest susceptibility, $b_{i}>b_{j}$ when $i<j$. Hence, $b_{1}$ is the largest infection rate constant while $b_{\mathrm{n}}$ is the smallest. As before, each $b_{i}$ is positive.

To ensure that the model represents a more realistic population, notice that it now has a birth rate constant, $\beta$, and a per-capita death rate constant, $\mu$, with the following proportions of births going into each respective class:

$$
p_{1}, p_{2}, p_{3}, \ldots, p_{n} \text {, and } p_{R}
$$

( $p_{R}$ will refer to the proportion of individuals born into the removed class and - thus - have immunity).

It is important to consider that $\mu$ affects all population groups equally; this is because it represents deaths not due to the disease (all disease-related deaths simply become part of the removed group). Note here that $1 / \mu$ signifies the average lifespan of healthy individuals in the population [5] and that the sum of all the proportions of births going into each class is one:

$$
\sum_{k=1}^{n} p_{k}+p_{R}=1 .
$$

\section{Features of an SIR Model of n Susceptibility Classes}

The total population size, which we will call $K$, is the summation of the individuals in the different susceptibility classes, the infectious class, and the removed class, giving the total dynamic population as:

$$
K^{\prime}=S_{1}^{\prime}+S_{2}^{\prime}+S_{3}^{\prime}+\cdots+S_{n}^{\prime}+I^{\prime}+R^{\prime},
$$

which simplifies to

$$
K^{\prime}=\beta-\mu K
$$

This differential equation yields the following solution $K(t)$, of which the limit can be taken as time goes to infinity, giving the approached population size, $\beta / \mu$ :

$$
\lim _{t \rightarrow \infty} K(t)=\lim _{t \rightarrow \infty}\left(\frac{\beta}{\mu}+C e^{-\mu t}\right)=\frac{\beta}{\mu} .
$$

Here, $C=K_{0}-\beta / \mu$, where $\mathrm{K}_{0}$ is the initial population size.

Another feature of an SIR model of $n$ susceptibility classes is that the model has two biologically-relevant equilibria: a disease-free equilibrium and an endemic equilibrium. A disease-free equilibrium denotes a state in which the number of infected individuals approaches zero, indicating that there is no disease present in the 
population of study [5]. The disease-free equilibrium for this model is found by setting all of the derivatives and $I$ equal to zero and solving for the remaining variables, producing the following equilibrium:

$$
\left(S_{1}, S_{2}, S_{3}, \ldots S_{n}, I, R\right)=\left(\frac{\beta p_{1}}{\mu}, \frac{\beta p_{2}}{\mu}, \frac{\beta p_{3}}{\mu}, \ldots \frac{\beta p_{n}}{\mu}, 0, \frac{\beta p_{R}}{\mu}\right) .
$$

The endemic equilibrium, on the other hand, represents a state in which the infections are persistent, but not persistent enough to cause an epidemic [5]. However, to determine if the disease will die out or not, we only need to determine if the number of infections, $I$, will approach 0 , or, equivalently, if the disease-free equilibrium is asymptotically stable. This is done by deriving what is known as the basic reproductive number of the disease, or $R_{0}$ for short. For an epidemic model, $R_{0}$ "represents the average number of secondary infections caused by each infective over the course of the infection" [1, pg. 290]. Theoretically, $R_{0}$ is representative of the threshold value between a disease-free state and an endemic state. If $R_{0}$ is less than one, then the disease-free equilibrium is stable; whereas if $R_{0}$ is greater than one, then the disease-free equilibrium is unstable, leading to an endemic disease. Determining which of these cases occurs can be done using the following theorem:

Theorem 1. The threshold value between a disease-free and an endemic state in (2) is described by:

$$
\mathrm{R}_{0}=\frac{\beta}{\mu}\left(\frac{\sum_{k=1}^{n} b_{k} p_{k}}{\mu+\alpha}\right)
$$

If $R_{0}>1$, then the disease will become endemic in the population; otherwise, if $R_{0}<1$, then the population will eventually become disease-free.

The proof of this theorem is presented in the Appendix (section 1).

It is important to note here that this theorem, and the theorems mentioned later in this manuscript, prove local stability of the equilibria, but the simulations suggest global stability.

\section{AN SIR MODEL OF THREE SUSCEPTIBILITY CLASSES}

In nature, the number of susceptibility classes varies from one population to the next and are dependent on the type of disease, but for the purpose of illustrating the behavior of the model, an SIR model of three susceptibility classes will be used:

$$
\begin{gathered}
S_{1}^{\prime}=-b_{1} S_{1} I+p_{1} \beta-\mu S_{1} \\
S_{2}^{\prime}=-b_{2} S_{2} I+p_{2} \beta-\mu S_{2} \\
S_{3}^{\prime}=-b_{3} S_{3} I+p_{3} \beta-\mu S_{3} \\
I^{\prime}=\left(\sum_{k=1}^{3} b_{k} S_{k}-\alpha-\mu\right) I \\
R^{\prime}=\alpha I+p_{R} \beta-\mu R .
\end{gathered}
$$

This model was originally intended to serve as a method of studying the behavior of influenza, which has a recovery period of a few days to two weeks [8]. Hence, $\alpha=1$ week for our simulations. $\beta$ and $\mu$ are also assigned specific values with the units of per week per 1000 people: $\beta=0.2415$ [6], and $\mu=0.0001421$ [3]. The remaining parameter values were selected through simulation work; namely, what appeared to be the most feasible cases for a realistic disease spread.

\section{VACCINATIONS}

With a limited number of available vaccinations, the individuals with the highest susceptibility to infection should be vaccinated first because highly susceptible individuals hold the most potential to contract and spread a disease. As a result of vaccination efforts, these individuals are taken out from the highest susceptibility class and placed into the removed group, revoking their role as active agents of disease spread. Such efforts will greatly reduce the impact of a disease by either diminishing the outbreak numbers or avoiding an outbreak outright.

This paper considers two possible methods of vaccination: point and interval vaccination. 


\section{Point Vaccination Scheme}

A point vaccination scheme (PVS) is administered at exactly time $\tau$ and in a single distribution cycle. This means that all the individuals suffering from the highest susceptibility to disease will be vaccinated in an instant, ideally preventing further spread of the disease by placing such individuals into the removed class. The model of such a scheme is the same as the SIR model of $n$ susceptibility classes; however, at time $\tau$, the conditions of $S_{1}(t)$ and $R(t)$ will be reset as follows:

$$
\begin{gathered}
S_{1}(\tau)=0 \\
R(\tau)=\lim _{t \rightarrow \tau_{-}}\left(S_{1}(t)+R(t)\right) .
\end{gathered}
$$

This reset occurs to satisfy the spontaneous nature of PVS. It affirms that with the administration of the vaccine at time $\tau$, all of the highly susceptible individuals will be classified as removed individuals.

From a public health standpoint, one may be curious as to the best time to administer such a vaccine. Ideally, it is administered when $S_{1}$ is at its highest. In many cases, this means the vaccine should be administered as early as possible, as illustrated by the following theorem:

Theorem 2. The optimal vaccination time for PVS occurs at time zero, given biologically realistic parameters.

The proof of this theorem is presented in the Appendix (section 2).

Using the model in (4), and an initial population of 1000, the disease spikes when no vaccine is administered and $R_{0}>1$; and the disease does not spike when a vaccine is administered and is effective in removing enough susceptible individuals to inhibit the disease spread. When the vaccine is effective, it entails that the disease has been successfully suppressed (Figure 1).

Figure 1. The Effectiveness of PVS.
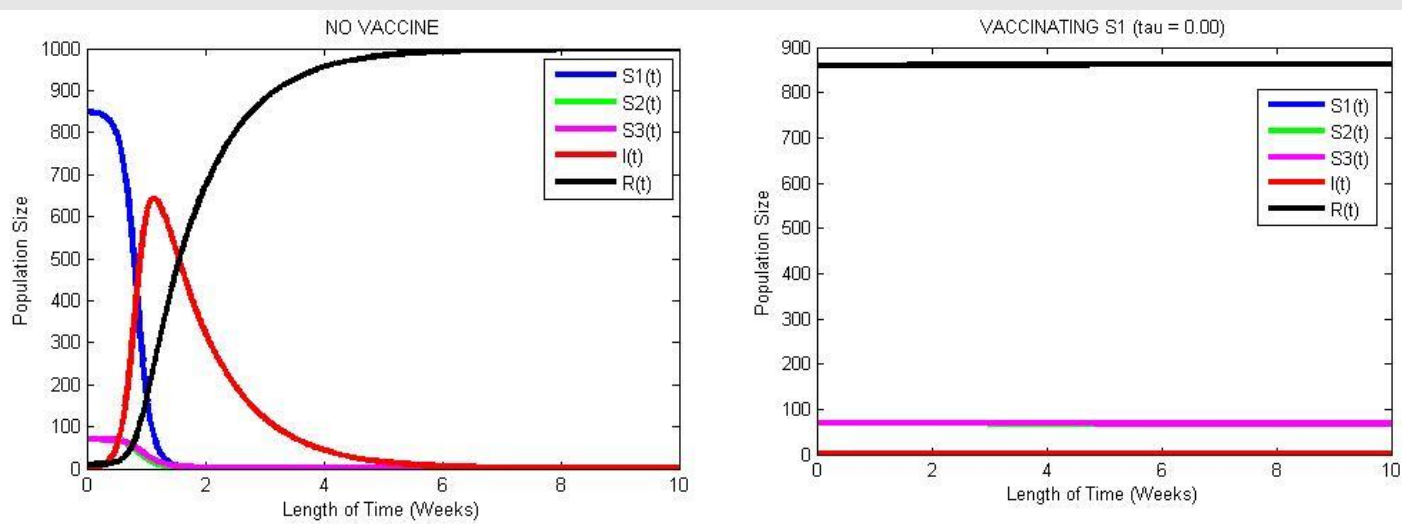

Parameters: $\alpha=1, \beta=0.2415, \mu=0.0001421, b_{1}=0.010, b_{2}=0.007, b_{3}=0.005, p_{1}=0.85, p_{2}=0.07, p_{3}=0.07, p_{R}=0.01$

Note 1: From this figure and onwards, the legend will be located vertically on the right of the graphs.

However, the disease is not necessarily eliminated from the population if any new births are placed in $S_{1}$, since the vaccine is administered only once. This allows the disease to potentially rebound and still become endemic, but it may take a long time before doing so, depending on how fast $\mathrm{S}_{1}$ is replenished.

Because Theorem 2 only holds for biologically realistic parameter values, one may find that $S_{1}(t)$ may be maximized at some other $t$ value besides zero if non-realistic parameter values are used. In Figure 2, $S_{1}$ is maximized elsewhere other than at time zero. This is because, while all the other parameters values have realistic values assigned to them, the birth constant, $\beta$, is set at 2000 births per week. Since the initial population size is 1000,2000 births per week boasts a $200 \%$ increase in the initial population in the first week. Obviously, this does not reflect biological realism because such a population swell is not realistic, as humans do not reproduce at such a high rate. This is why it is emphasized that Theorem 2 only works under practical conditions. 
Figure 2. The Ineffectiveness of PVS.
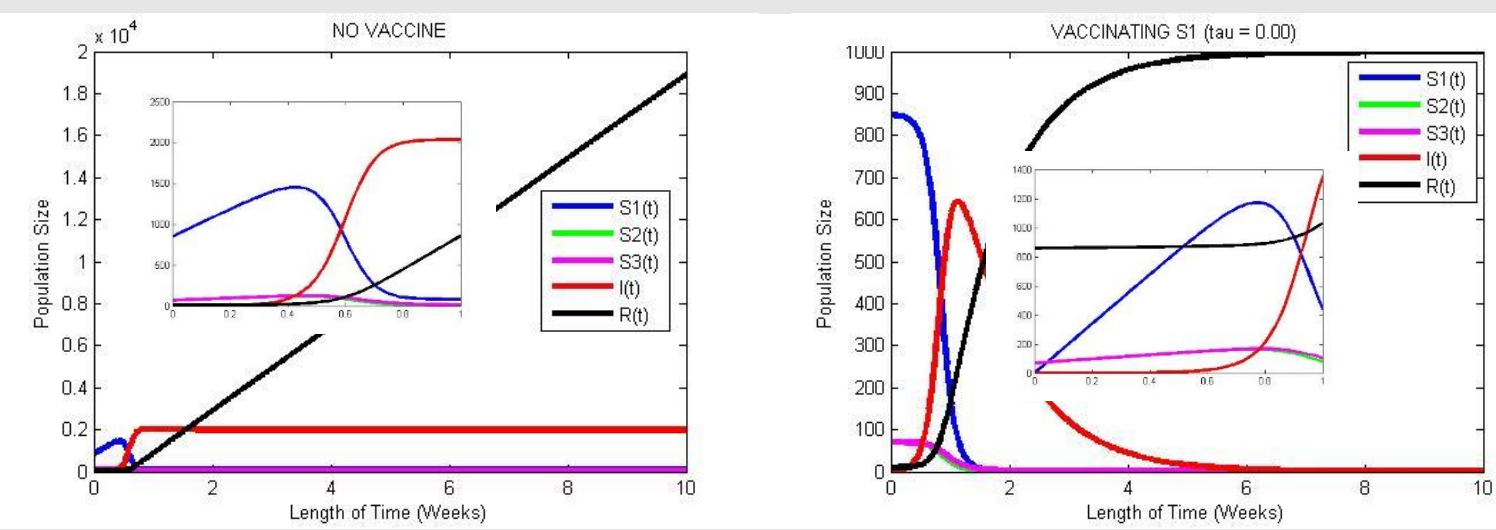

Parameters: $\alpha=1, \beta=2000, \mu=0.0001421, b_{1}=0.050, b_{2}=0.0006, b_{3}=0.0005, p_{1}=0.33, p_{2}=0.33, p_{3}=0.33, p_{R}=0.01$

Note 2: The smaller, zoomed in graphs are presented for illustration purposes to demonstrate that $S_{1}$ is maximized elsewhere. The time range for these graphs is set to 1 week for this purpose.

\section{Interval Vaccination Scheme}

An interval vaccination scheme (IVS) is a vaccine that is administered starting at time $\tau$ and is re-administered over an interval of length $H$. Like a PVS, an IVS is also administered in one distribution cycle. Similarly, the model of IVS is that of the SIR model of $n$ susceptibility classes; however, the equations for $S^{\prime}{ }_{1}(t)$ and $R^{\prime}(\mathrm{t})$ are reset between the time interval $[\tau, \tau+H]$ as follows:

$$
\begin{gathered}
S_{1}^{\prime}(t)=-b_{1} S_{1} I+p_{1} \beta-\mu S_{1}-\min \left(\frac{1}{H} S_{1}(\tau), S_{1}(t)\right) \\
R^{\prime}(t)=\alpha I+p_{R} \beta-\mu R+\min \left(\frac{1}{H} S_{1}(\tau), S_{1}(t)\right) .
\end{gathered}
$$

Here, $H$ represents the length of the vaccination time interval; in an IVS, the vaccines are administered uniformly over the entire interval. The reset asserts that within the time interval $[\tau, \tau+H]$, a constant proportion of the highly susceptible individuals will be vaccinated at each instant, allowing IVS to offer more realism in its distribution because not all the highly susceptible individuals can be vaccinated at a single point in time. Meanwhile, the remaining highly susceptible individuals may eventually become infected or may remain susceptible, given that there is a continuous flow of births during the vaccination interval and not everyone is vaccinated at once. Otherwise, everyone that is available to be vaccinated at the beginning of the interval either will be vaccinated or will get infected. Once the reset has progressed beyond the time interval $[\tau, \tau+H]$, the system will revert back to following the standard SIR model of $n$ susceptibility classes.

Using biologically realistic parameters, it turns out that an IVS can work in reducing the number of infected to zero over a timescale of 10 weeks (Figure 3). Again, it seems that vaccinating these individuals can result in a disease-free state, if studied over a short time scale; if the system is studied over a longer time scale, $R_{0}$ is the determining factor for the stability of the disease-free equilibrium.

The key properties of an IVS are that the highly susceptible individuals are marked for vaccination at time $\tau$, and only these individuals have the chance of being vaccinated. Referring back to Figure 3, it is noticeable that the largest pool of highly susceptible individuals occurs at time zero in most cases, as proven in Theorem 2 , and, since that is the case, then - logically - an IVS is also best distributed at time zero. This leads to the following conjecture (it has not yet been proven, but logical and simulation evidence suggest it will be true in most cases): 
Figure 3. The Effectiveness of IVS.
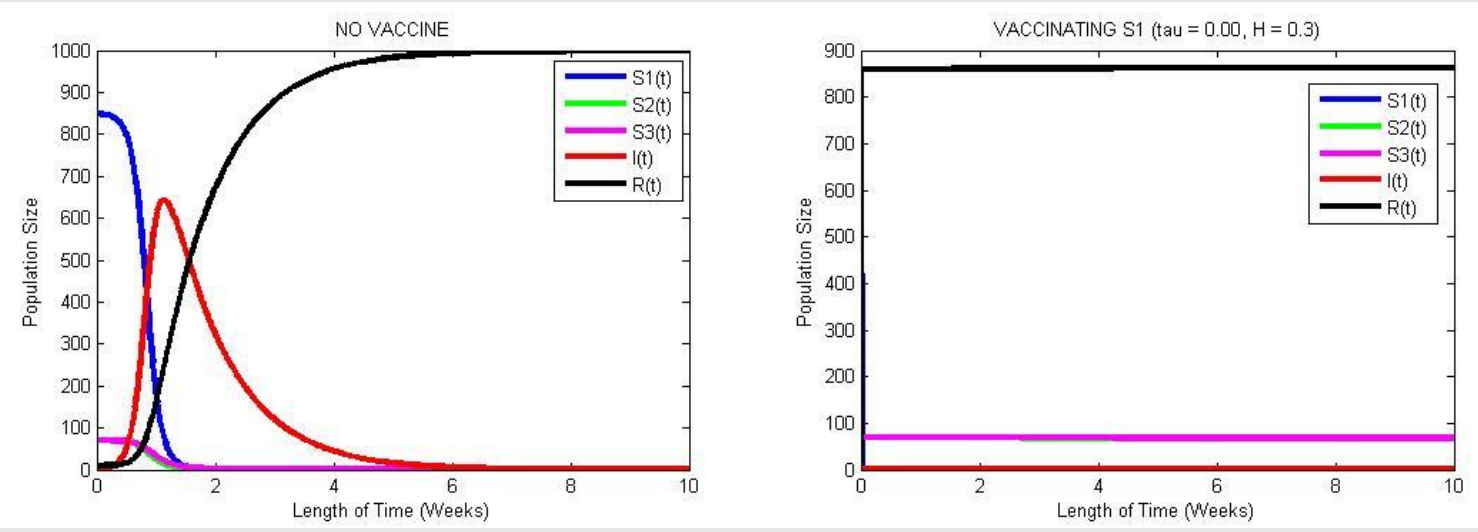

Parameters: $\alpha=1, \beta=0.2415, \mu=0.0001421, b_{1}=0.010, b_{2}=0.007, b_{3}=0.005, p_{1}=0.85, p_{2}=0.07, p_{3}=0.07, p_{R}=0.0$

Conjecture 1. The IVS is most effective when vaccines are distributed starting at time zero, given biologically realistic parameters.

The evidence of this conjecture is provided in the Appendix (section 3).

\section{REGAINING SUSCEPTIBLITY}

There have been incidents reported in which an administered vaccine offers little aid in the prevention of disease. This is because some vaccines lack the necessary potential to prevent an outbreak, leaving those already vaccinated susceptible to primary and recurring infection. For instance, individuals who receive Bacillus Calmette-Guérin vaccines at a young age are not always protected from tuberculosis [4].

Similarly, in the case of the flu, an individual who has received vaccination or has gained immunity may regain susceptibility. The flu is an infection of the respiratory tract caused by different influenza viruses, defining the flu as a class of viruses that similarly affect the human body. There is no singular and definable "flu virus," and the class of related viruses possess a variable genetic makeup. Because of the different strains of viruses, individuals who previously received flu vaccines can become infected with a different strain of the flu - behavior that would appear as a "recurring [epidemic]" [1, pg. 298]. It is important to note that these individuals never receive the same virus twice; it is always a mutated strain of virus that caused a previous infection or a virus from a different strain.

Almost every year, $5-20 \%$ of the population in the United States is infected with the seasonal flu [11]. The virus that causes seasonal flu changes a little bit each year, but the changes are small and people have some resistance to the virus [9]. In some years, the flu virus changes drastically and results in another epidemic, in which the individuals who have been previously vaccinated for the seasonal flu become susceptible to this new strain.

Another strain similar to the one described above is the H1N1 strain. In 2009, the H1N1 strain infected 22 million people in the United States, causing an estimate of 98,000 H1N1-related hospitalizations and 3,900 H1N1-related deaths [12]. Because both the seasonal flu strain and H1N1 flu strain originated from an ancestor virus, the latent period of both influenza strains coincide. This collision results in individuals who are highly susceptible to both strains. Additionally, although an individual may be more immune to one flu virus over another, he or she can come down with the flu again, disguised by a difference in strain.

\section{Average Rate Model}

To illustrate an instance of regained susceptibility, the initial SIR model of $n$ susceptibility classes in (2) is modified through the addition of a portion of removed individuals into the highest susceptibility class as follows: 


$$
\begin{gathered}
S_{1}^{\prime}=-b_{1} S_{1} I+p_{1} \beta-\mu S_{1}+c R \\
S_{2}^{\prime}=-b_{2} S_{2} I+p_{2} \beta-\mu S_{2} \\
S_{3}^{\prime}=-b_{3} S_{3} I+p_{3} \beta-\mu S_{3} \\
\cdots \\
S_{n}^{\prime}=-b_{n} S_{n} I+p_{n} \beta-\mu S_{n} \\
I^{\prime}=\left(\sum_{k=1}^{n} b_{k} S_{k}-\alpha-\mu\right) I \\
R^{\prime}=\alpha I+p_{R} \beta-\mu R-c R .
\end{gathered}
$$

Here, $c$ represents the rate at which an individual regains susceptibility on average, where $1 / c$ denotes the average length of time an individual remains immune before regaining susceptibility to infection. $c$ is assumed positive for this model.

As seen with an SIR model of $n$ susceptibility classes in (2), there are both disease-free and endemic equilibria, with the stability of the disease-free equilibrium determined by a basic reproductive number. For this model, the disease-free equilibrium is:

$$
\left(S_{1}, S_{2}, S_{3}, \ldots, S_{n}, I, R\right)=\left(\frac{\beta p_{1}+c R^{*}}{\mu}, \frac{\beta p_{2}}{\mu}, \frac{\beta p_{3}}{\mu}, \ldots, \frac{\beta p_{n}}{\mu}, 0, R^{*}\right)
$$

where $R^{*}=\frac{\beta p_{R}}{\mu+c}$.

The $R_{0}$ value can be found using the same Jacobian technique used in the proof of Theorem 1 (see the Appendix, Section 1). The basic reproductive number for this model is denoted as:

$$
R_{0}=\frac{\beta}{\mu}\left(\frac{\sum_{k=1}^{n} b_{k} p_{k}}{\mu+\alpha}+\frac{b_{1} p_{1} c}{(\mu+\alpha)(\mu+c)}\right) .
$$

This $R_{0}$ value is extremely similar to the one derived in Theorem 1, except for the addition of the term $\frac{b_{1} p_{1} c}{(\mu+\alpha)(\mu+c)}$. Notice that $\frac{b_{1} p_{1} c}{(\mu+\alpha)(\mu+c)}$ is bound to be small and will minimally impact the basic reproductive number as consequence. This finding was made through running numerous simulations for various values of the parameters in the additional term; it was found that the only impact on $R_{0}$ that would be significant is if that term was large enough to cause $R_{0}$ to go above the threshold value of one, pending it was less than one with respect to the original model in (2). This could happen if $c$ was large, in conjunction with a high $b_{1}$ and a high $p_{1}$, and the original value of $R_{0}$ in (2) was already fairly close to one. On the other hand, if these parameters are low, then the term involving $c$ will also be small, entailing that the impact of regaining susceptibility will be negligible. Thus, depending on a system's reproductive number when immunity is effectively permanent, the introduction of possible susceptibility rehash can hold little or great sway on the end state of a disease.

\section{Fixed Rate Model}

The representation of $c$ as an average is problematic, as $c$ is derived from an exponential distribution [1]. On average, individuals are taken from the removed class around time $c$ and are then subsequently placed into the highest susceptibility group. But, an average is not all telling. There is potential for an individual to be removed at a time far displaced from that of time $c$, since that time for immunity can vary anywhere from zero to infinity. For example, if $c=0.1$, then - on average - a removed individual is taken out from the removed class approximately after 10 weeks. Realistically, such an individual cannot be taken out from the removed class at a time that extends beyond that individual's lifetime. However, since - in an average rate model - the time for immunity can range anywhere from zero to an infinite period, it is possible that an individual will regain susceptibility past his or her lifetime. An applicable solution to this issue is a model of fixed rate, where individuals regain susceptibility after a known, fixed amount of time: 


$$
\begin{gathered}
S_{1}^{\prime}=-b_{1} S_{1} I+p_{1} \beta-\mu S_{1}+\left(\alpha I(t-c)+p_{R} \beta-\mu R(t-c)\right) \\
S_{2}^{\prime}=-b_{2} S_{2} I+p_{2} \beta-\mu S_{2} \\
S_{3}^{\prime}=-b_{3} S_{3} I+p_{3} \beta-\mu S_{3} \\
\cdots \\
S_{n}^{\prime}=-b_{n} S_{n} I+p_{n} \beta-\mu S_{n} \\
I^{\prime}=\left(\sum_{k=1}^{n} b_{k} S_{k}-\alpha-\mu\right) I \\
R^{\prime}=\alpha I+p_{R} \beta-\mu R-\left(\alpha I(t-c)+p_{R} \beta-\mu R(t-c)\right),
\end{gathered}
$$

where $c$ represents the exact time an individual maintains immunity.

Again, our goal for this system will be to analyze the stability of the disease-free equilibrium, which is the same as for the original model (2). It turns out that, unlike in (7), where the rate $c$ has some impact on the spread of the disease, $c$ does not impact the stability of the disease-free state at all in this model, as the following theorem states:

Theorem 3. The threshold value between a disease-free and an endemic state in (10) is described by:

$$
\mathrm{R}_{0}=\frac{\beta}{\mu}\left(\frac{\sum_{k=1}^{n} b_{k} p_{k}}{\mu+\alpha}\right) .
$$

The proof for this theorem is in the Appendix (section 4).

The basic reproductive number for the fixed rate model is identical to the basic reproductive number found for an SIR model of $n$ susceptibility classes (8). This denotes that the delay terms do not affect the basic reproductive number of a fixed rate model.

\section{Comparison of Average Rate Model and Fixed Rate Model}

Recall that in both models of regained susceptibility, the parameter, $c$, represents the rate at which a removed individual regains susceptibility. For the analysis of both models, a 4 week immunity period will be studied. This equates to $c$ being valued at 0.25 and 4.0 for the average rate and fixed rate models, respectively. As done previously, we will assume three susceptibility classes are present (4).

Interestingly, both models have almost the same end behavior at 52 weeks, suggesting that the two approach the same endemic equilibrium (Figure 4A). Dissimilarly, though, the average rate model approaches a fixed endemic equilibrium, whereas the fixed rate model suffers continued oscillations in the class populations. Further inspection of the numerical data in Table 1 provides different end behavior for the two models. Thus, to fully determine the end behavior , the models need to be studied over a longer time.

At 100 weeks, both models again seem to have the same end behavior, once more suggesting that they approach the same endemic equilibrium (Figure 4B). However, the model depicting the average rate still approaches a fixed endemic equilibrium, while the model depicting a fixed rate experiences less severe oscillations and apparently approaches an equilibrium close to that of the average rate model (Table 1).

At 1000 weeks, the end behavior of the two models is almost, but not exactly, the same (Figure $\mathbf{4 C}$ and Table 1). 
Figure 4. Average Rate Model and Fixed Rate Model at Various Lengths of Time.

A)
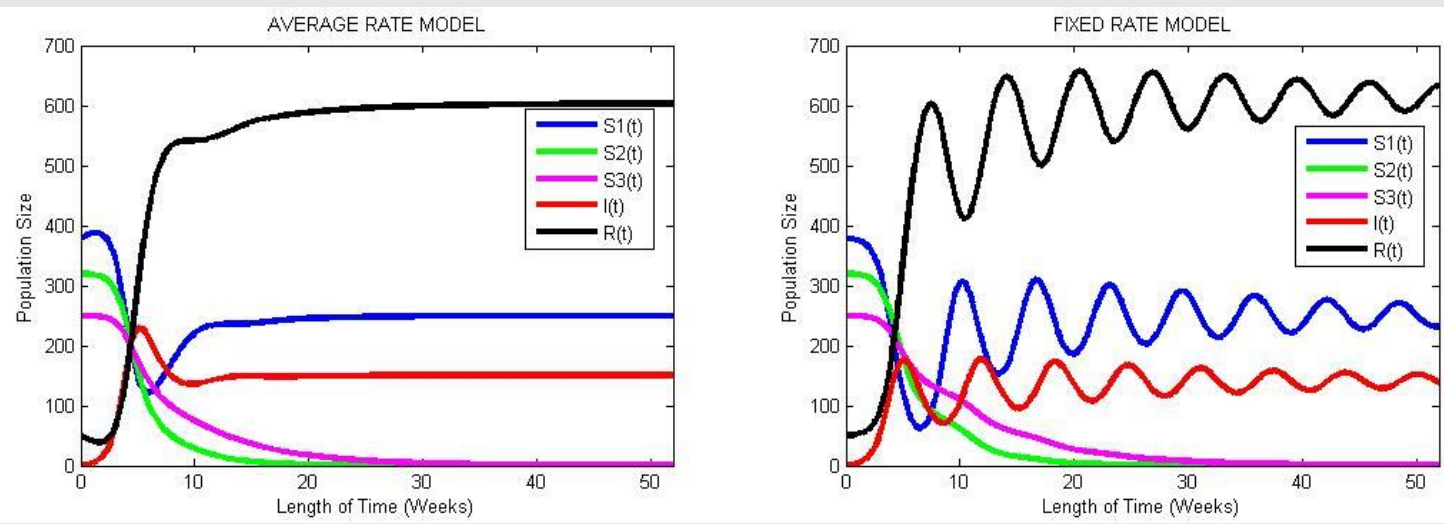

B)
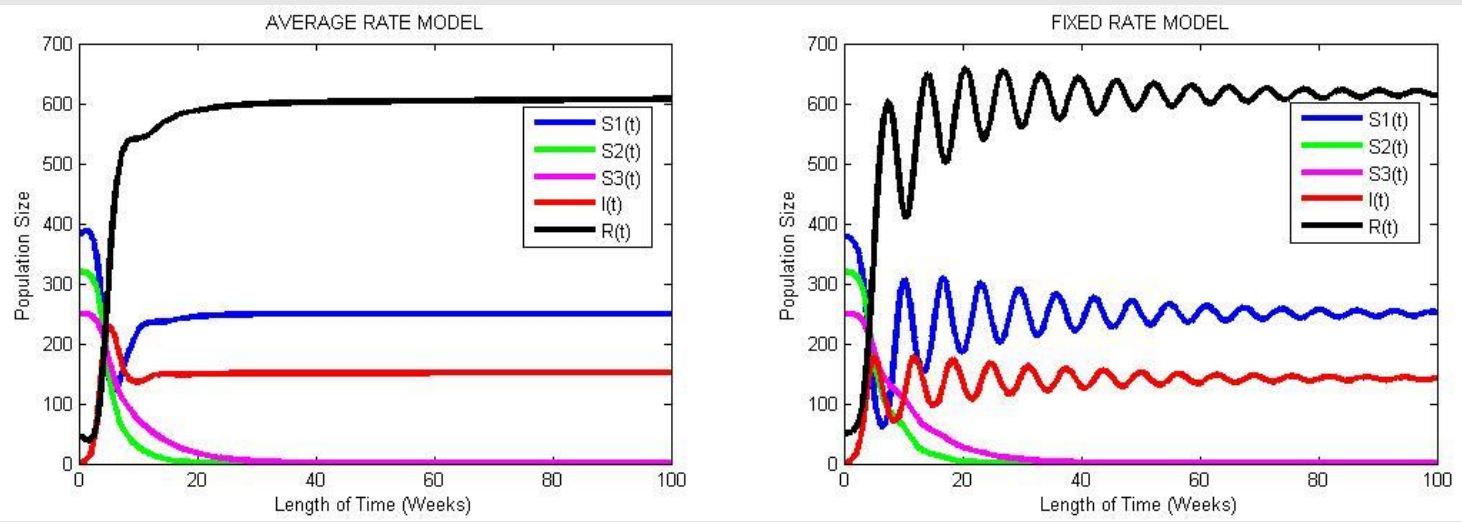

C)
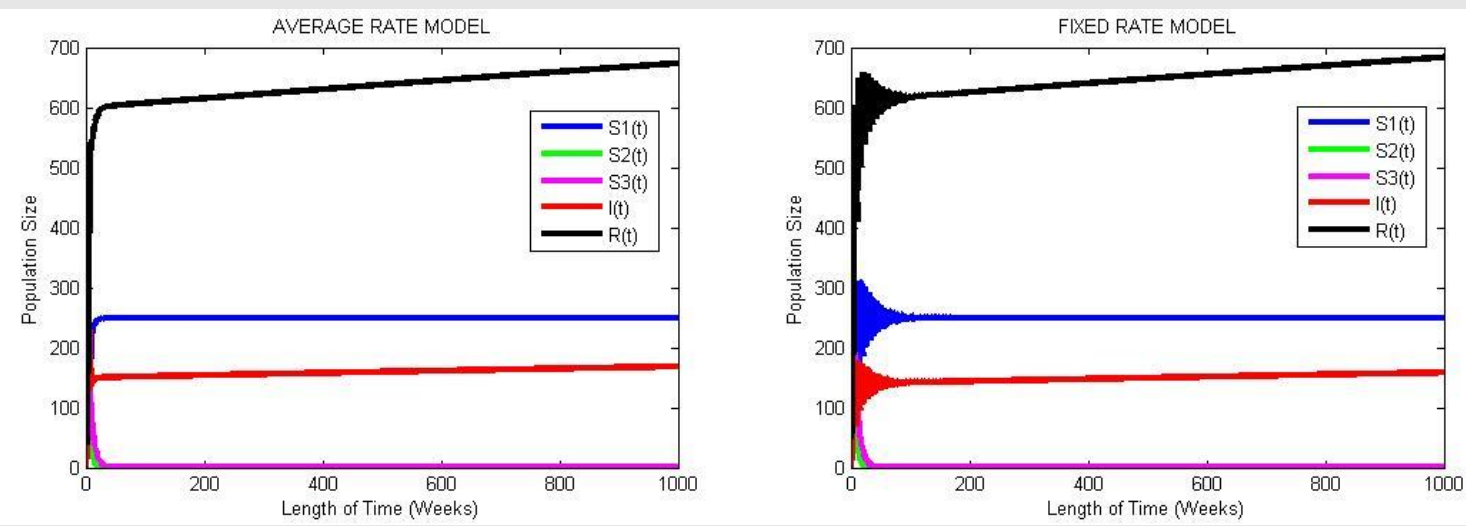

Parameters: $\alpha=1, \beta=0.2415, \mu=0.0001421, b_{1}=0.004, b_{2}=0.002, b_{3}=0.001, p_{1}=0.38, p_{2}=0.32, p_{3}=0.25, p_{R}=0.01$ 
Table 1. Average Rate and Fixed rate at Various Lengths of Time.

\begin{tabular}{|l|l|c|c|c|c|l|l|}
\hline $\mathbf{T}$ (Weeks) & Model Type & $\mathbf{S}_{\mathbf{1}}$ & $\mathbf{S}_{\mathbf{2}}$ & $\mathbf{S}_{\mathbf{3}}$ & $\mathbf{I}$ & $\mathbf{R}$ & Population Size \\
\hline \multirow{2}{*}{$\mathbf{5 2}$} & Average Rate & 249.8085 & 0.2559 & 0.5365 & 151.0435 & 603.5054 & $\mathbf{1 0 0 5 . 1}$ \\
\cline { 2 - 8 } & Fixed rate & 256.6548 & 0.2754 & 0.6089 & 149.0352 & 598.9700 & $\mathbf{1 0 0 5 . 5}$ \\
\hline \multirow{2}{*}{$\mathbf{1 0 0}$} & Average Rate & 249.8414 & 0.2542 & 0.3973 & 151.9975 & 607.3793 & $\mathbf{1 0 0 9 . 9}$ \\
\cline { 2 - 8 } & Fixed rate & 250.0352 & 0.2723 & 0.4253 & 140.4167 & 619.1121 & $\mathbf{1 0 1 0 . 3}$ \\
\hline \multirow{2}{*}{$\mathbf{1 0 0 0}$} & Average Rate & 249.8570 & 0.2292 & 0.3581 & 168.5654 & 673.6510 & $\mathbf{1 0 9 2 . 7}$ \\
\cline { 2 - 8 } & Fixed Rate & 249.8458 & 0.2436 & 0.3806 & 158.6008 & 683.9347 & $\mathbf{1 0 9 3 . 0}$ \\
\hline
\end{tabular}

Parameters: $\alpha=1, \beta=0.2415, \mu=0.0001421, b_{1}=0.004, b_{2}=0.002, b_{3}=0.001, p_{1}=0.38, p_{2}=0.32, p_{3}=0.25, p_{R}=0.01$

Both models exhibit the same end behavior, and since there is insignificant difference in the behavior of these curves, the long term behavior of a disease can be studied using either of the two models. However if a disease is to be studied over a shorter time scale, both models will have to be analyzed as they both exhibit different behaviors in the beginning.

\section{CONCLUSION}

In addition to the presentation of an SIR model of $n$ susceptibility classes, this research most conclusively provides arguments attesting to the merit of early vaccination distribution. Such theorems hold that both the point and interval vaccination schemes are best distributed at time zero, as the pools of susceptible individuals are largest then. Distributing vaccinations to only individuals of the highest susceptibility class ensures the most efficient allocation of resources and the most marginal reduction in the spread of disease, especially if the number of people in this group is high. Thus, to quell a disease, if vaccines are to be exclusively applied to individuals of the highest susceptibility, vaccination efforts are to be expended as early as possible in order for the efforts to be most substantial in disease prevention.

To enhance the study of a disease spread, two additional models introduce the possibility of regained susceptibility: using an average time or a fixed time of regaining susceptibility. Such models allow for removed individuals to re-cycle into a susceptibility class, either as a result of ineffective vaccines or of genetic variants in a strand of a disease. The impact of doing so appears to be minimal at best, and nonexistent in the case of fixed time, but it is important to consider this effect if the basic reproductive number, without considering a regaining of susceptibility, is very close to 1 in the case of average time.

Overall, a much more thorough understanding of disease spread has been gained and may be utilized in controlling environmental diseases with resources limited to the highly susceptible individuals. In the future, we will work on proving Conjecture 1, which our numerical and graphical evidence suggests is true. Furthermore, a mortality delay model will be developed, a model whose purpose will be to remove infected individuals from the infectious class, indicating a disease-related death. Such a model will further enhance the study of disease spread.

\section{ACKNOWLEGDEMENTS}

This research would not be possible without the thoughtful supervision of Dr. Anthony DeLegge and the funding provided by the Benedictine University College of Science Summer Research Program, directed by Dr. Lee Ann Smith. 


\section{APPENDIX}

\section{Section 1}

Proof of Theorem 1: As stated earlier, to determine a formula for $R_{0}$ for (2), we need to study the stability of the disease-free equilibrium. This involves a standard Jacobian analysis. The Jacobian matrix of an SIR model of $n$ susceptibility classes at the point of the disease-free equilibrium is as follows:

$$
\left[\begin{array}{ccccccc}
-\mu & 0 & 0 & 0 & 0 & -\frac{b_{1} p_{1} \beta}{\mu} & 0 \\
0 & -\mu & 0 & 0 & 0 & -\frac{b_{2} p_{2} \beta}{\mu} & 0 \\
0 & 0 & -\mu & 0 & 0 & -\frac{b_{3} p_{3} \beta}{\mu} & 0 \\
\cdots & \cdots & \cdots & \cdots & \cdots & \cdots & \cdots \\
0 & 0 & 0 & 0 & -\mu & -\frac{b_{n} p_{n} \beta}{\mu} & 0 \\
0 & 0 & 0 & 0 & 0 & \frac{\beta}{\mu}\left(\sum_{k=1}^{n} b_{k} p_{k}\right)-\alpha-\mu & 0 \\
0 & 0 & 0 & 0 & 0 & \alpha & -\mu
\end{array}\right]
$$

Through simple row operations, the general Jacobian matrix of this model is triangular, meaning its eigenvalues can just be read off of the diagonal. Thus, the eigenvalues of the above matrix are:

$$
\begin{gathered}
\lambda_{1}=\frac{\beta}{\mu}\left(\sum_{k=1}^{n} b_{k} p_{k}\right)-\alpha-\mu \\
\text { and } \\
\lambda_{2}=-\mu .
\end{gathered}
$$

Since $-\mu$ is guaranteed to be negative, we only need to check the other eigenvalue for stability of the diseasefree equilibrium:

$$
\frac{\beta}{\mu}\left(\sum_{k=1}^{n} b_{k} p_{k}\right)-\alpha-\mu .
$$

This quantity must be negative for the system to be asymptotically stable in its disease-free state. Thus through algebraic manipulation, the following inequality surfaces:

$$
R_{0}=\frac{\beta}{\mu}\left(\frac{\sum_{k=1}^{n} b_{k} p_{k}}{\mu+\alpha}\right)<1 .
$$

If the prior inequality holds true, then the disease-free equilibrium is asymptotically stable; otherwise, if the inequality is false, then the disease-free equilibrium is unstable, meaning the disease will be endemic in the population [2]. This expression is representative of the threshold between a disease-free and an endemic state.

This completes the proof of Theorem 1.

\section{Section 2}

Proof of Theorem 2 : To prove this theorem, two related lemmas will be established.

Lemma 1. $S_{1}$ is decreasing at time zero under biologically realistic parameters.

Proof of Lemma 1: From the model in (2), it is known that $S_{1}^{\prime}=-b_{1} S_{1} I+p_{1} \beta-\mu S_{1}$. All of the terms in $S_{1}^{\prime}$ are negative except for $p_{1} \beta$. Since $p_{1}$ is bounded by one and multiplied by $\beta$, the term $p_{1} \beta$ is the only term that has the potential to make $S_{1}^{\prime}$ positive and, thus, change the behavior of $S_{1}(t)$ from decreasing to increasing. If it can be proven that $S_{1}(t)$ is decreasing at time zero, then it can be assumed that $t=0$ is a local maximum of $S_{1}(t)$, as no points before $t=0$ are of consequence and the function is known to be decreasing immediately after time 
zero. In proving this, it is important to note that the initial conditions of the system set $I(0)=1$ and $S_{1}(0)=$ $p_{1} K_{0}-1$. Plugging these values into $S_{1}^{\prime}$ gives

$$
S_{1}^{\prime}(0)=-b_{1}\left(p_{1} K_{0}-1\right)(1)+p_{1} \beta-\mu\left(p_{1} K_{0}-1\right) .
$$

In order for $S_{1}(t)$ to be decreasing at time zero, the following inequality must hold:

$$
\frac{p_{1} \beta}{b_{1}+\mu}<p_{1} K_{0}-1
$$

which arranges to

$$
\frac{p_{1} \beta}{p_{1}+\mu}<S_{1}(0)
$$

In a population inflicted by disease, we do not expect births to occur incessantly, nor do we expect the births to greatly contribute to the highly susceptible class. Thus, so long as the birth rate and/or the proportion of births that are highly susceptible are sufficiently small, then this inequality will be satisfied. Therefore, $S_{1}$ decreases at time zero, given biologically realistic parameter values.

Lemma 2. The absolute maximum value of $S_{1}$ occurs at $t=0$ given biologically realistic parameters.

Proof of Lemma 2: From knowledge of calculus and the first derivative test, any critical point of a function occurs when its derivative is zero. So, should any other value of $S_{1}$ exceed that of $S_{1}(0)$, it would be at a point where

$$
S_{1}^{\prime}(t)=-b_{1} S_{1} I+p_{1} \beta-\mu S_{1}=0,
$$

which simplifies through algebraic manipulation to

$$
S_{1}=\frac{p_{1} \beta}{p_{1} I+\mu}
$$

This is to be recognized as similar to the inequality above, denoting the conditions under which $S_{1}(t)$ is decreasing at time zero. In order for the value of $S_{1}(0)$ to remain above the value of $S_{1}(t)$ at a local maximum, then it must hold that

$$
p_{1} K_{0}-1>\frac{p_{1} \beta}{b_{1} I+\mu}
$$

Solving for $I$, the above inequality yields

$$
I>\frac{p_{1} \beta}{b_{1}\left(p_{1} K_{0}-1\right)}-\frac{\mu}{b_{1}}
$$

which condenses to

$$
I>\frac{p_{1} \beta-\mu\left(p_{1} K_{0}-1\right)}{b_{1}\left(p_{1} K_{0}-1\right)}
$$

and rearranges to

$$
I>\frac{\left.p_{1}\left(\beta-\mu K_{0}\right)+\mu\right)}{b_{1}\left(p_{1} K_{0}-1\right)} .
$$

When the resulting inequality surfaces whenever $S_{1}$ has a critical point, then the absolute maximum of $S_{1}(t)$ will be at time zero. In addition, if the expression $p_{1} K_{0}-1$ is not greater than zero, the population in study will be too small to actually be relevant, so it is assumed that

$$
p_{1} K_{0}-1>0 \text {. }
$$

One way to satisfy the inequality is to make the right side negative. For this to occur, $p_{1}\left(\beta-\mu K_{0}\right)+\mu$ in (11) has to be less than zero, which simplifies to 


$$
\frac{1}{p_{1}}<K_{0}-\frac{\beta}{\mu}
$$

Recall that $K_{0}$ is the initial population size and $\beta / \mu$ is the approached population size. Since $\beta / \mu>K_{0}$ if the initial population is increasing over time, the inequality, $\frac{1}{p_{1}}<K_{0}-\frac{\beta}{\mu}$, is proven false. However, if the population is decreasing, and $p_{1}$ is not very small, then the inequality will be true. That being said, if $S_{1}(t)$ is initially decreasing, then $S_{1}(t)$ will be less than its initial amount at any time.

The original inequality in (11) can also be satisfied pending that $\beta-\mu K_{0}$ is positive, meaning the population size is increasing over time, and $p_{1}$ and $\mu$ are small, positive numbers, assuming that the population does not grow too much over its initial size. If $\mu$ is not small, it will not just affect the highest susceptibility class, but there will also be many more deaths in the overall population, since $\mu$ is present in all the groups. Inclusively, this will lead to a slower spread of the infection, which could result in a rebound of $S_{1}$. Similarly, if $p_{1}$ is very large (that is, close to 1), then that means there will be too many individuals in the highest susceptibility class, and the initial population in the highly susceptible class can potentially be replaced with numerous fresh births after a wave of infections if $\beta$ is high enough, again allowing $S_{1}$ to rebound. Realistically, we do not expect a fast-growing population during a disease spread, nor do we expect a high natural death rate or birth rate. Thus, given biologically reasonable conditions, the absolute maximum of $S_{1}(t)$ should occur at $t=0$.

Since Lemma 2 implies that the most available highly susceptible individuals in $S_{1}$ will be at $t=0$, thus, a PVS is best administered at time zero.

This completes the proof of Theorem 2 .

\section{Section 3}

Evidence of Conjecture: If the highest susceptibility class is vaccinated at a time other than zero, such as when $\tau=0.1$ or $\tau=0.01$, it means that a smaller pool of the most susceptible individuals will be vaccinated than if the vaccines were administered at $\tau=0$ by Lemma 1 (Appendix, Section 2). Lemma 1 also guarantees that the largest number of susceptibles will occur at time 0 , so starting vaccines at any other time will mean that a smaller pool is present. Thus, $\tau=0$ should still be the most effective time for vaccination distribution.

Table 2. Testing the Effectiveness of $\tau$ when $\tau \neq 0$ and $H=0.30$.

\begin{tabular}{|c|c|c|c|c|c|c|c|}
\hline T (Weeks) & T (Weeks) & $S_{1}$ & $S_{2}$ & $S_{3}$ & I & $\mathbf{R}$ & \# of Vaccinated Individuals \\
\hline \multirow{2}{*}{0.00} & 10 & 0.3601 & 0.3717 & 0.5223 & 0.0514 & 999.6710 & \multirow{2}{*}{248.9255} \\
\hline & 52 & 2.8855 & 2.8971 & 3.0469 & 0.0000 & 996.3035 & \\
\hline \multirow{2}{*}{0.02} & 10 & 0.3610 & 0.3726 & 0.5229 & 0.0506 & 999.6694 & \multirow{2}{*}{248.7378} \\
\hline & 52 & 2.8864 & 2.8980 & 3.0475 & 0.0000 & 996.3011 & \\
\hline \multirow{2}{*}{0.06} & 10 & 0.3628 & 0.3744 & 0.5236 & 0.0491 & 999.6665 & \multirow{2}{*}{248.1382} \\
\hline & 52 & 2.8882 & 2.8998 & 3.0482 & 0.0000 & 996.2967 & \\
\hline \multirow{2}{*}{0.08} & 10 & 0.3637 & 0.3753 & 0.5236 & 0.0484 & 999.6654 & \multirow{2}{*}{247.6710} \\
\hline & 52 & 2.8891 & 2.9007 & 3.0483 & 0.0000 & 996.2949 & \\
\hline \multirow{2}{*}{0.10} & 10 & 0.3646 & 0.3762 & 0.5233 & 0.0477 & 999.6646 & \multirow{2}{*}{247.0391} \\
\hline & 52 & 2.8900 & 2.9016 & 3.0480 & 0.0000 & 996.2934 & \\
\hline \multirow{2}{*}{0.20} & 10 & 0.3686 & 0.3801 & 0.5130 & 0.0446 & 999.6701 & \multirow{2}{*}{238.6380} \\
\hline & 52 & 2.8940 & 2.9055 & 3.0377 & 0.0000 & 996.2957 & \\
\hline
\end{tabular}




\begin{tabular}{|l|l|l|l|l|l|l|l|}
\hline \multirow{2}{*}{$\mathbf{0 . 3 0}$} & $\mathbf{1 0}$ & 0.3701 & 0.3812 & 0.4724 & 0.0434 & 999.7089 & \multirow{2}{*}{$\mathbf{2 0 5 . 3 4 5 7}$} \\
\cline { 2 - 6 } & $\mathbf{5 2}$ & 2.8955 & 2.9067 & 2.9978 & 0.0000 & 996.3330 & \\
\hline \multirow{2}{*}{$\mathbf{0 . 4 0}$} & $\mathbf{1 0}$ & 0.3648 & 0.3758 & 0.4221 & 0.0471 & 999.7666 & \multirow{2}{*}{$\mathbf{1 1 8 . 6 0 0 4}$} \\
\cline { 2 - 6 } & $\mathbf{5 2}$ & 2.8902 & 2.9012 & 2.9474 & 0.0000 & 996.3941 & \\
\hline
\end{tabular}

Parameters: $\alpha=1, \beta=0.2415, \mu=0.0001421, b_{1}=0.030, b_{2}=0.025, b_{3}=0.015, p_{1}=0.25, p_{2}=0.25, p_{3}=0.25, p_{R}=0.25$

In Table 2, the effectiveness of an IVS is monitored over the course of both 10 week and 52 week periods, and it seems that starting vaccinations at a time slightly after time zero is still effective in suppressing the disease. However, as $\tau$ increases, the number of effectively vaccinated individuals decreases and further diverges from the simulation beginning.

Recall the purpose of the vaccination schemes: to reduce the number of highly susceptible individuals and - as consequence - the number of infected individuals and to increase the number of individuals in the removed class. Now, if an IVS is administered to the highest susceptibility class at a time other than zero, then the maximum number of the individuals in the infective class will increase, more individuals will remain in the highest susceptibility class, and fewer individuals will be removed from susceptibility (Table 2).

Figure 5. Testing the Effectiveness of an IVS when $\tau \neq 0$.
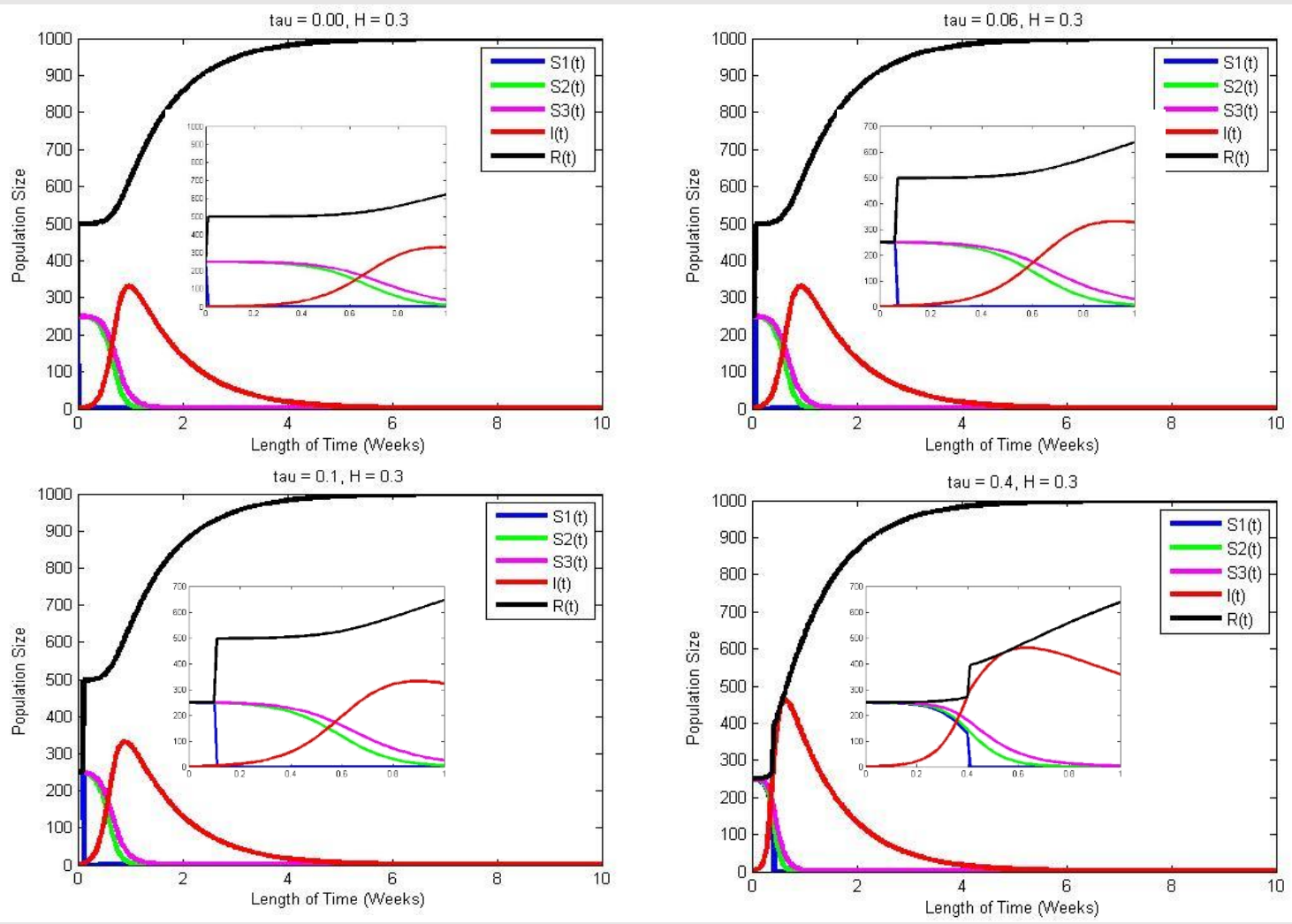

Parameters: $\alpha=1, \beta=0.2415, \mu=0.0001421, b_{1}=0.030, b_{2}=0.025, b_{3}=0.015, p_{1}=0.25, p_{2}=0.25, p_{3}=0.25, p_{R}=0.25$

Note 3: The smaller, zoomed in graphs are presented for illustrations purposes to demonstrate disease distribution near time zero. The time range for these graphs is set to 1 week for this purpose.

That said, it is to be recognized that the IVS should be effective so long as the vaccine is distributed reasonably close to the start of the outbreak. Furthermore, with a limited quantity of vaccinations, it is more pragmatic to begin vaccine distribution at the time of initial outbreak, thus maximizing the potential help of the inoculations 
(Figure 5). Therefore, the graphical and numerical evidence presented strongly suggests our conjecture is correct, and other simulation work done also seems to confirm this conjecture.

This completes the evidence of the Conjecture.

\section{Section 4}

Proof of Theorem 3: Since the fixed rate model (10) has delay terms, $\mu R(t-c)$ and $\alpha I(t-c)$, we need to account for this when linearizing the system [7].

According to the following characteristic equation [7], solving for lambda yields the eigenvalues for this system:

$$
\left|J_{0}+e^{-\lambda \tau_{1}} J_{\tau_{1}}-\lambda \mathbf{I}\right|=0 .
$$

In this equation, $J_{0}$ is the Jacobian matrix from the Appendix (section 1) while $J_{\tau_{1}}$ is the Jacobian matrix found with respect to the differential delay equations. This is done by setting all non-delay terms equal to 0 and computing the relevant partial derivatives with respect to the delay terms. Note that $\mathbf{I}$ in the characteristic equation is the identity matrix.

By substituting the disease-free equilibrium (3) into each matrix and setting the characteristic expression for the fixed rate model equal to zero, lambda is solved for to obtain the following eigenvalue as the determining factor for stability for the disease-free state (all other eigenvalues are negative):

$$
\lambda=-\frac{\sum_{k=1}^{n}-b_{k} p_{k} \beta+\alpha \mu+\mu^{2}}{\mu} .
$$

As before, we require all eigenvalues to be negative to ensure the disease-free state is asymptotically stable.

After setting this eigenvalue equal to zero and rearranging it, the following basic reproductive number is obtained as well as a condition for stability:

$$
R_{0}=\frac{\beta}{\mu} \frac{\sum_{k=1}^{n} b_{k} p_{k}}{\mu+\alpha}<1
$$

We recognize this as the same $R_{0}$ as that of the original model (2).

This completes the proof of Theorem 3. 


\section{REFERENCES}

[1] F. Brauer, C. Castillo-Chavez. Mathematical Models in Population Biology and Epidemiology. New York, NY; Springer-Verlog, 2010, pp. 275-337.

[2] F. Brauer, C. Castillo-Chavez. Mathematical Models for Communicable Diseases. Philadelphia; SIAM, 2013, pp. 32-37.

[3] D.L. Hoyert, J. Xu. Deaths: preliminary data for 2011. Natl Vital Stat Rep, 2011 [cited 28 May 2014], 61(6). Available from: http://www.cdc.gov/nchs/data/nvsr/nvsr61/nvsr61_06

[4] P.K. Giri, G.K. Khuller. Is intranasal vaccination a feasible solution for tuberculosis? Expert review of vaccines, 2008, 7(9): 1341-56.

[5] A. Korobeinikov. 2009. Global Properties of SIR and SEIR Epidemic Models with Multiple Parallel Infectious Stages. Bulletin of Mathematical Biology. 71: 75-83.

[6] J.A. Martin et al. Births: final data for 2012. Natl Vital Stat Rep, 2013 [cited 28 May 2014], 62(9). Available from http://www.cdc.gov/nchs/data/nvsr/nvsr62/nvsr62_09

[7] M. R. Roussel. Delay-differential equations. 2005. Available from: http://webcache.googleusercontent.com/search?q=cache:rIDIEGBBQRUJ:people.uleth.ca/ roussel/nl d/delay.pdf $+\& \mathrm{~cd}=1 \& \mathrm{hl}=$ en $\& \mathrm{ct}=\mathrm{clnk} \& \mathrm{gl}=\mathrm{us}$

[8] Flu Symptoms \& Severity [Internet]. Center for Disease Control and Prevention; c2015 [cited 10 April 2016]. Available from: http://www.cdc.gov/flu/about/disease/symptoms.htm

[9] H1N1 flu and seasonal flu: differences and similarities [Internet]. Department of Health; c2010 [cited 28 November 2013]. Available from http://www.health.ny.gov/publications/7227/

[10] Infectious diseases [Internet]. Center for Strategic International Studies; c2013 [cited 28 November 2013]. Available from http://www.smartglobalhealth.org/issues/entry/infectious-diseases

[11] Seasonal flu [Internet]. Center for Disease Control and Prevention; c2013 [cited 28 November 2013]. Available from: http://www.cdc.gov/flu/about/qa/disease.htm

[12] Updated CDC estimates of 2009 H1N1 influenza cases, hospitalizations and deaths in the United States, April 2009 - April 10, 2010 [Internet]. Centers for Disease Control and Prevention; c2010 [cited 28 November 2013]. Available from: http://www.cdc.gov/h1n1flu/estimates_2009_h1n1.htm 